\title{
Tumor renal con invasión hepática: aportación de un nuevo caso y revisión de la literatura
}

\author{
Quicios Dorado C, Mayayo Dehesa T, Nuño Vázquez-Gaza J*, García Teruel D*, \\ López Buenadicha A*, Díez Nicolás V.
}

Servicio de Urología. *Servicio de Cirugía General y Digestivo. Hospital Ramón y Cajal, Madrid.

Actas Urol Esp. 2007;31(5):541-547

\section{RESUMEN}

TUMOR RENAL CON INVASIÓN HEPÁTICA: APORTACIÓN DE UN NUEVO CASO Y REVISIÓN DE LA LITERATURA

El carcinoma de células renales (CCR) con invasión directa de órganos adyacentes es un hallazgo infrecuente y de mal pronóstico en el que el único tratamiento potencialmente efectivo es la cirugía radical. Se presenta el caso de una mujer con gran masa renal y afectación hepática y diafragmática sometida a tratamiento quirúrgico, y se realiza revisión de la literatura.

Palabras clave: Carcinoma de células renales (CCR). Factores pronósticos. Cirugía radical.

\section{ABSTRACT}

RENAL CELL CARCINOMA WITH LIVER EXTENSION: A REPORT OF A NEW CASE AND LITERATURE REVIEW

Locally advanced renal cell carcinoma (RCC) with involvement to adjacent organs is uncommon and the prognosis is poor. Radical surgery remains the only effective treatment. We report the case of a woman with RCC and direct liver extension who was surgically treated. A literature review is made.

Keywords: Renal cell cancer (RCC). Prognosis factors. Radical surgery.

A ctualmente el diagnóstico temprano del carcinoma de células renales (CCR) se realiza gracias al uso extendido de técnicas de imagen para evaluar otros procesos médicos.

El CCR con invasión directa de órganos adyacentes sucede aproximadamente en un $10 \%$ de todos los CCR. Su presencia aumenta el estadio patológico, disminuye la probabilidad de curación quirúrgica y representa un signo de mal pronóstico ${ }^{1}$. Se estima que la supervivencia a 3 años de estos pacientes es menor del $5 \%{ }^{2}$.

A pesar de los avances en terapias sistémicas adyuvantes para el CCR, la extirpación quirúrgica sigue siendo la mejor opción para lograr una mayor supervivencia a largo plazo en pacientes seleccionados con CCR y afectación hepática por contigüidad.

\section{CASO CLÍNICO}

Mujer de 65 años sin antecedentes personales de interés que ingresa por cuadro de fiebre elevada sin foco aparente de 1 semana de evolución, motivo por el que se realiza TC toracoabdominal observando masa de gran tamaño $(9 \times 8 \times 7 \mathrm{~cm})$ en polo superior renal derecho, en intimo contacto con el hígado (Figs. 1 y 2). No refiere otra sintomatología salvo dolor e impotencia funcional en miembro inferior izquierdo de meses de evolución en seguimiento por Servicio de Neurocirugía y con diagnóstico, tras realización de TC de columna y Resonancia Magnética Nuclear (RMN) de patología degenerativa, estenosis de canal L4-L5 y espondiloartrosis. No presenta síndrome constitucional ni dolor abdominal. 

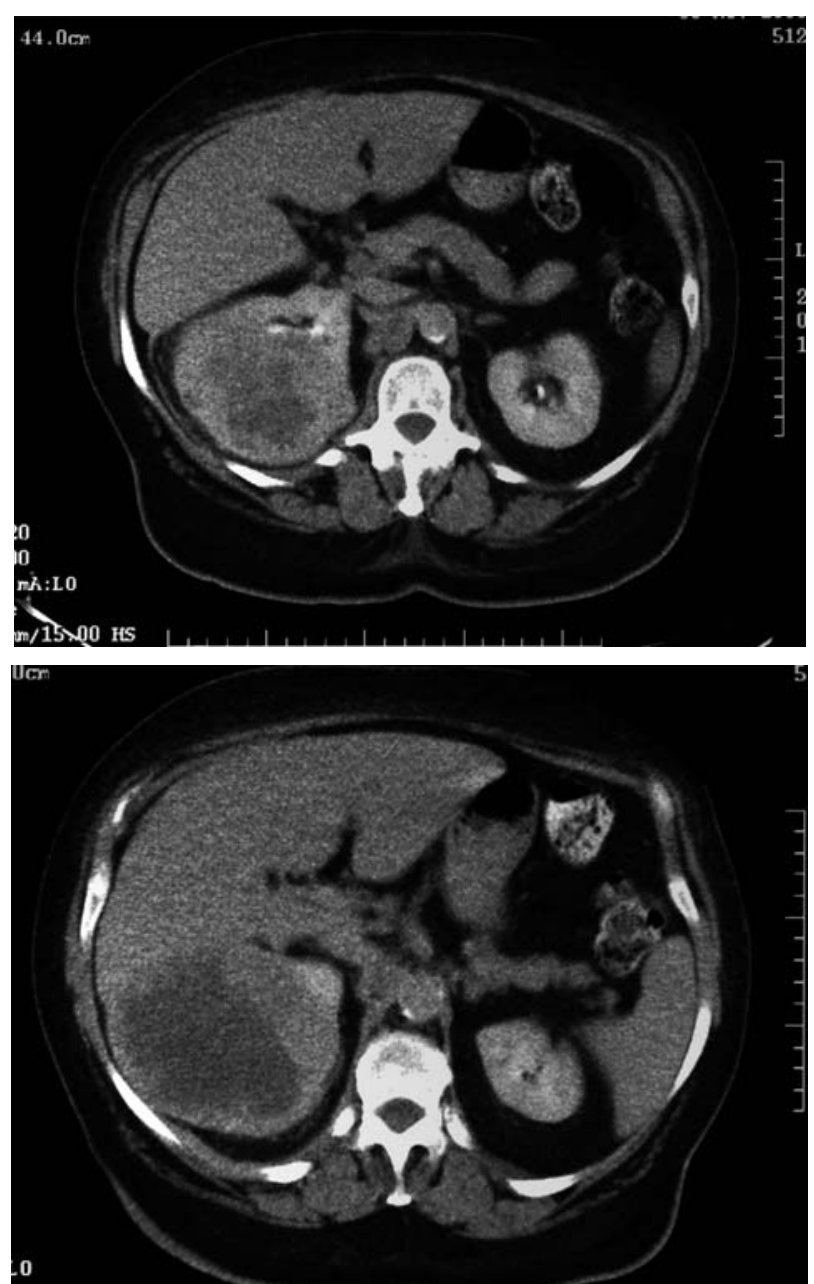

FIGURAS 1 y 2. TAC: Masa en polo superior renal derecho en intimo contacto con el hígado.

En la exploración física presenta abdomen blando y depresible, globuloso, sin palpación de masas ni megalias. No dolor a la exploración. Puño-percusión renal bilateral negativa.

Analíticamente presenta bioquímica sin alteraciones con perfil hepático, proteínas totales y calcio en rango normal. Hemograma con hemoglobina de 9,4 g/dL, y 22.500 leucocitos (N: 72,9\%). Hemostasia sin hallazgos. Hemocultivos estériles y urocultivos $<10.000 \mathrm{UFC} / \mathrm{ml}$.

Se instaura tratamiento antibiótico de amplio espectro y antitérmico a pesar de lo cual presenta picos febriles diarios con repercusión clínica en forma de astenia.

Ante los hallazgos en TC se decide realizar RMN (Figs. 3 y 4) confirmando masa sólida en polo superior derecho de 14X10X10 cm de diámetro, con gran heterogeneidad de señal y necro- sis en su interior. La lesión se extiende en sentido ascendente hacia el hígado con signos de infiltración hepática. La vena renal derecha y vena cava se encuentran libres. Existen adenopatías retrocavas, derrame pleural y engrosamiento diafragmático.

Dada la afectación hepática se comenta el caso con el servicio de Cirugía General y Digestivo acordando realizar TAC Multicorte para delimitar con mayor exactitud el nivel de afectación de dicho órgano y planificación de la intervención quirúrgica,
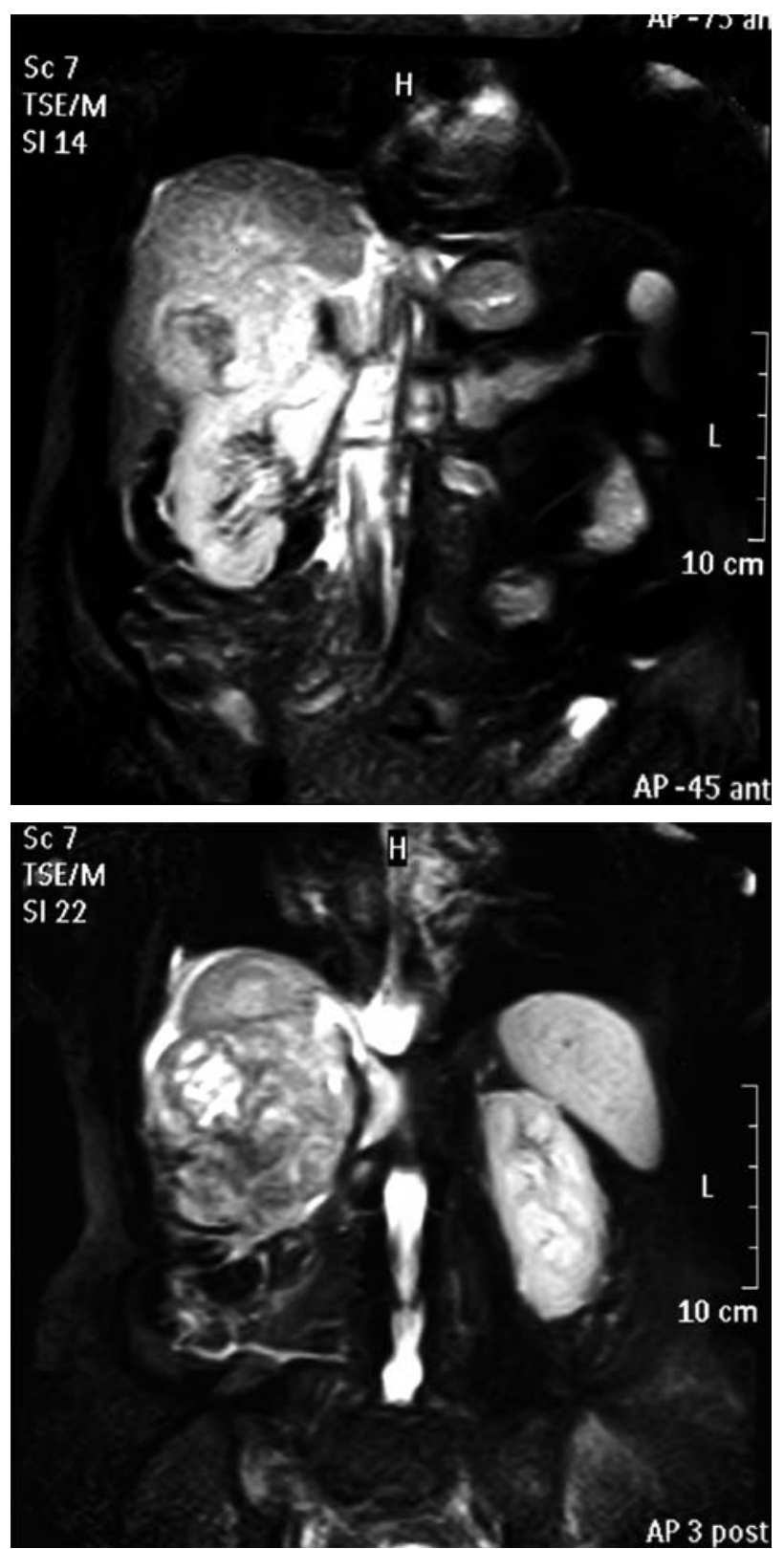

FIGURAS 3 y 4. RMN: masa con necrosis en su interior. Venas renal derecha y cava libres. 
apreciando masa heterogénea de aspecto necrótico y diámetro máximo de $11 \mathrm{~cm}$. en mitad superior de riñón derecho sin plano de separación de los segmentos postero-superiores de lóbulo hepático derecho, existiendo afectación por contigüidad de los mismos (Fig. 5).

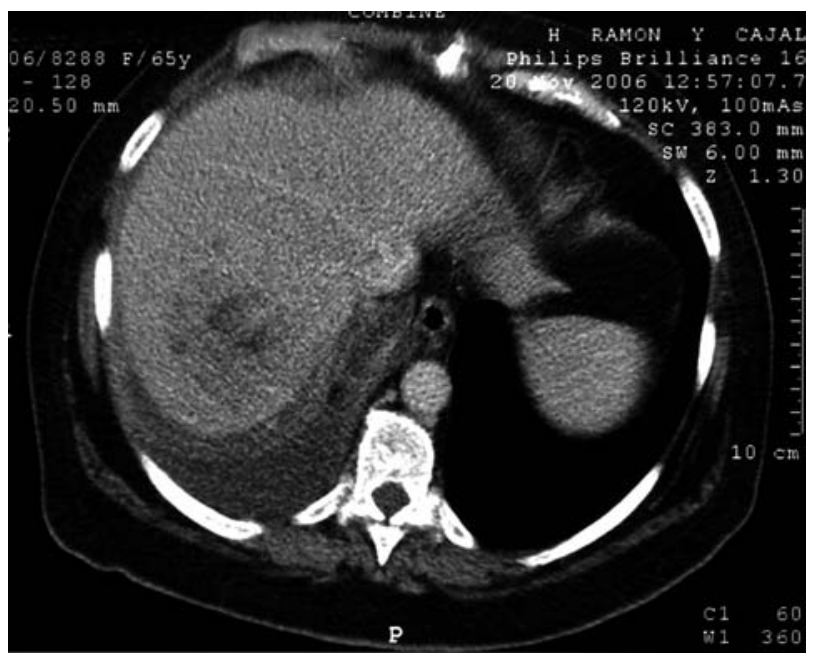

FIGURA 5. TAC MULTICORTE: afectación por contigüidad de lóbulo hepático derecho.

Previa embolización renal se realiza intervención quirúrgica a través de laparotomía media supra e infraumbilical. La exploración muestra tumoración renal que infiltra el hígado (segmento VII) y diafragma, apreciando adenopatías patológicas en hilio renal y líquido peritoneal libre sin evidencia de implantes peritoneales. Se toman muestras de líquido peritoneal para cultivo, citología y gram con resultado negativo. Se practica nefrectomía radical derecha incluyendo en bloque el segmento hepático VII, adrenal derecha y porción de diafragma. Colecistectomía y linfadenectomía.

El análisis anatomopatológico revela un cáncer de células renales tipo convencional de alto grado citológico (grado 4 de Fuhrman), con focos de transformación sarcomatoide y extensas áreas de necrosis tumoral. Dos nódulos satélites en superficie peritoneal de diafragma y metástasis en un ganglio linfático del hilio renal. No afectación del sistema venoso ni excretor renal ni de la glándula suprarrenal. Estadio pT4G3N1 según la UICC.

Tras un postoperatorio satisfactorio con disminución progresiva de leucocitosis comienza tratamiento quimioterápico oral con inhibidores de la angiogénesis. En el segundo TC de control realizado dos meses después de la intervención se evidencia derrame pleural derecho con lesiones nodulares pleurales basales y posteriores. La citología del líquido pleural es compatible con adenocarcinoma.

\section{DISCUSIÓN}

La afectación hepática secundaria a carcinoma de células renales (CCR) puede ocurrir por diseminación hematógena ó por extensión directa del tumor.

Las metástasis intrahepáticas son más frecuentes que la extensión local de la enferme$\operatorname{dad}^{2,3}$, ocurren aproximadamente en el $20 \%$ de los pacientes con CCR y son menos frecuentes que otras localizaciones como pulmón (70\%), ganglios linfáticos (50\%) o hueso (40\%). Además presentan peor pronóstico ya que la mayoría de las veces son múltiples y se asocian con metástasis en otros lugares. Sólo en $2-4 \%$ de los casos las metástasis hepáticas de CCR son localizadas o solitarias $^{2-4}$.

La extensión de un tumor renal a un órgano adyacente al riñón diferente de la glándula suprarrenal ipsilateral sucede aproximadamente en un $10 \%$ de todos los $\mathrm{CCR}^{2,5,6}$, y aunque un tumor renal de grandes proporciones puede comprimir el parénquima hepático, la invasión directa rara vez ocurre ${ }^{2,7,8}$. Estos pacientes presentan alto riesgo de recurrencia de la enfermedad con tasas que oscilan entre 50-85\% dependiendo del estado tumoral (T) y afectación de ganglios linfáticos $(\mathrm{N})^{8}$.

Numerosas masas renales permanecen asintomáticas y no palpables hasta que alcanzan un estadio evolutivo avanzado lo que determina que algunos pacientes presenten un tumor primario voluminoso que invade estructuras circundantes, ocasionando dolor secundario a la invasión de la pared abdominal posterior, raíces nerviosas ó músculos paraespinosos. También pueden ocasionar hemorragia y raras veces la triada de dolor lumbar, hematuria macroscópica y masa abdominal palpable. En un $20 \%$ de los casos existen sindromes paraneoplásicos (HTA, poliglobulia, síndrome de Stauffer, hipercalcemia, etc.) debido a la liberación de sustancias por el tumor ó por 
las células que combaten a éste. Habitualmente desparecen tras la cirugía, y, en caso contrario, se debe sospechar metástasis o recidiva local.

En el caso que nos ocupa la paciente había permanecido completamente asintomática presentándose clínicamente con fiebre, anemia y leucocitosis. La fiebre en el CCR suele ser resistente a tratamiento antibiótico, no tiene repercusión sobre el pronóstico y desaparece tras la nefrectomía, como sucedió en este caso. Se produce como reacción ante necrosis tumoral, infecciones intratumorales ó por secreción de sustancias pirógenas. Se han identificado inmunomoduladores como IL-6, IL-1 e interferones que estimulan la síntesis de proteínas de fase aguda en el hígado, producen leucocitosis, aumento de la velocidad de sedimentación (VSG) y fiebre ${ }^{7}$.

El hecho de que numerosas masas renales permanezcan asintomáticas favorece su diagnóstico al realizar pruebas de imagen por otros problemas médicos. Ante su sospecha la primera técnica de imagen a realizar es el $\mathrm{TC}^{2,9}$. Se debe realizar RMN en aquellos pacientes con cáncer localmente avanzado, si existe posible compromiso venoso, insuficiencia renal o alergia al contraste. Es la técnica de elección para estudiar invasión tumoral de estructuras circundantes y en la evaluación y estadificación de trombos tumorales en el sistema venoso ${ }^{7}$.

Los factores pronósticos más importantes son los relacionados con el tumor: estadio histopatológico, tamaño tumoral, grado nuclear y subtipo histológico.

El estadio histopatológico es el factor pronóstico aislado más importante en pacientes con CCR. La magnitud de la enfermedad locorregional o sistémica en el momento del diagnóstico es el factor determinante principal de la evolución de esta enfermedad. Así, la invasión local de órganos adyacentes representa un signo de mal pronóstico, aumenta el estadio patológico y disminuye la probabilidad de curación quirúrgi$\mathrm{ca}^{1,2,7,10}$. DeKernion et al. ${ }^{10}$ informan que los pacientes con CCR y afectación de vísceras adyacentes por contigüidad tratados quirúrgicamente presentan una supervivencia menor del 5\% a 3 años, falleciendo la mayoría de los pacientes en $\operatorname{los} 2$ años siguientes ${ }^{10,11,12,13}$. El pronóstico de estos pacientes es peor que el de aquellos con metástasis hepáticas aisladas, con supervivencia del $11 \%$ a 3 años $2,4,7,14$.

La afectación de ganglios linfáticos supone un ensombrecimiento del pronóstico ${ }^{11}$ con supervivencia a 5 años de 5-30\% y a 10 años de 0-5\% $0^{7,15}$.

Varios autores afirman que tumores renales primarios con tamaño superior a $10 \mathrm{~cm}$. se asocian a peor pronóstico ${ }^{2,16}$. En un estudio realizado por Giuliani et al. ${ }^{16}$ se obtienen resultados de supervivencia de $84 \%$ en tumores menores de 5 $\mathrm{cm}$, de $50 \%$ en tumores de $5-10 \mathrm{~cm}$, y de $0-5 \%$ si superan los $10 \mathrm{~cm}$.

A mayor grado nuclear (Sistema de Fuhrman) peor pronóstico. Leibovich et al. ${ }^{17}$ plantean un algoritmo de predicción de supervivencia en pacientes con CCR metastático y afirman que los pacientes con grado nuclear de 4 tienen un 53\% más de posibilidades de morir que aquellos con tumor de bajo grado. También defienden que los tumores con áreas de necrosis tumoral se asocian con un 36\% más de probabilidades de muerte que aquellos sin necrosis ${ }^{17}$.

Dentro del subtipo histológico la presencia de diferenciación sarcomatoide se asocia con disminución significativa de la superviven$\mathrm{cia}^{2,7,11,17}$. Representan áreas mal diferenciadas de otros subtipos histológicos de CCR y no un tumor de origen independiente, observándose en $1-5 \%$ de CCR, por lo general del tipo convencional. Se caracteriza por patrón de crecimiento infiltrativo con comportamiento local y metastático agresivo. Bennet et al. ${ }^{2}$ realizan nefrectomía radical y hepatectomía parcial a 3 pacientes con CCR y afectación hepática por contigüidad. Dos de ellos presentaron diferenciación sarcomatoide en el resultado anatomopatológico con progresión de la enfermedad en 6 meses y supervivencia menor de 14 meses. Sin embargo, como se expone más adelante, existen casos de estas características descritos en la literatura sometidos a cirugía radical con aumento de la tasa de supervivencia.

A pesar de los últimos avances en terapias sistémicas para CCR metastático, la extirpación quirúrgica del tumor representa el único tratamiento potencialmente curativo de esta enferme$\operatorname{dad}^{2,6,11,13}$, siendo el objetivo la extirpación completa del tumor con márgenes quirúrgicos negati$\operatorname{vos}^{4,6,17}$. 
Aunque la tasa de respuesta (completa/parcial) a varios tratamientos sistémicos alternativos puede oscilar entre $10-35 \%{ }^{18,19}$, dado el pronóstico ominoso del CCR con extensión a órganos adyacentes, el tratamiento quirúrgico con resección hepática está indicado en pacientes seleccionados con afectación hepática limitada. A la hora de considerar esta cirugía es importante evaluar la función hepática del paciente, su estado general, la existencia de metástasis a distancia y el estado del hígado no afectado (enfermedades como cirrosis o ascitis aumentan la mortalidad contraindicando la hepatectomía) $)^{6,10}$.

Existen datos a favor de un aumento de supervivencia de los pacientes sometidos a nefrectomía radical más hepatectomía. Foster et al. ${ }^{20}$ revisan la supervivencia de pacientes sometidos a hepatectomía por metástasis tumorales, de los cuales 14 tenían tumor primario renal. Realiza seguimiento a 8 de estos últimos con supervivencia a 5 años por encima del $50 \%$. Kazugoshi et al. ${ }^{6}$ presentan 2 pacientes que tras nefrectomía y extirpación de segmentos VI y VII hepáticos se encuentran libres de enfermedad a 100 y 57 meses tras la cirugía. Otro estudio realizado en 4 pacientes presenta supervivencia global a 1 y 3 años tras la cirugía del $66 \%$ y $33 \%$ respectivamente $15,21,22$.

Es importante la resección completa del tumor con los órganos adyacentes afectados como demuestra deKernion et al. ${ }^{10}$, en cuya serie sólo el $12 \%$ de los pacientes con resección incompleta tumoral estaban vivos al año de la cirugía. La ausencia de márgenes negativos en la hepatectomía implica un peor pronóstico ${ }^{4,17}$.

A pesar del mal pronóstico de los CCR con extensión local y diferenciación sarcomatoide existen estudios que también demuestran mejoría de la supervivencia con la extirpación quirúrgica completa. J.A. Wong et $\mathrm{al}^{1}$ presentan un caso con extirpación renal, de glándula adrenal, segmentectomía hepática derecha, $10 \mathrm{~cm}$ de diafragma y lobectomía inferior derecha pulmonar con supervivencia mayor de 5 años. Del mismo modo S. Sakaguchi et al. ${ }^{23}$ informan una supervivencia libre de enfermedad mayor de 4 años postcirugía en un paciente de 35 años con CCR y diferenciación sarcomatoide tras reacción en bloque de riñón, glándula adrenal, lóbulo hepático y vena cava inferior.
Es importante recordar que actualmente existe una baja mortalidad quirúrgica $(<5 \%)$ y postoperatoria $(0-4 \%)$ con la realización de cirugía radical, respecto a épocas pasadas ${ }^{2,6}$.

Hasta ahora los tratamientos adyuvantes incluyendo radioterapia (RT), quimioterapia (QT) ó inmunoterapia no han mostrado resultados satisfactorios en CCR localmente avanzado 4,8,24. La QT intravenosa convencional no es efectiva y la RT sólo estaría indicada como tratamiento paliativo en pacientes con metástasis sistémi$\operatorname{cas}^{7,24}$.

En diversos estudios se obtuvieron resultados prometedores $^{8,25}$ en el tratamiento de CCR metastático estadio IV con el uso de IL-2 intravenosa ó subcutánea administrada sola o en combinación con IF- $\alpha$, alcanzando tasas de respuesta objetivas entre 19-31\%. Posteriormente, la combinación de citoquinas subcutáneas con QT intravenosa (5-FU) parecía reforzar su actividad antineoplásica logrando tasas de respuesta del $18-39 \% \%^{8,26,27}$.

Basándose en estos resultados Atzpodien et al. ${ }^{12}$ realizan un estudio clínico prospectivo randomizado para comparar la eficacia de IL-2, IF- $\alpha$ y 5-FU como tratamiento adyuvante frente a la observación, en 203 pacientes con CCR de alto riesgo (entre los que se encuentra pacientes con ganglios linfáticos positivos o extensión local de la enfermedad con afectación de órganos adyacentes) libres de enfermedad tras cirugía radical. Demostraron que los pacientes con CCR de alto riesgo no se benefician de inmunoterapia adyuvante postcirugía en cuanto a supervivencia libre de enfermedad y supervivencia global frente a la observación. Estos resultados fueron desalentadores sobre todo al compararlos con la eficacia demostrada en CCR metastático $8,26,27$.

Los inhibidores de la angiogénesis (SUNITINIB, SORAFENIB) son agentes emergentes e importantes en el tratamiento de CCR metastáti$\operatorname{co}^{28,29,30,31}$. Motzer et al. ${ }^{32}$ demuestran una mayor supervivencia libre de enfermedad, un aumento de la tasa de respuesta objetiva así como una mejoría en la calidad de vida de los pacientes tratados con Sunitinib en comparación con los tratados con IF- $\alpha$. Son necesarios más estudios referentes a esta nueva línea de tratamiento. 
A pesar de todo, en el manejo actual del CCR con afectación hepática por contigüidad, y siempre que el estado general del paciente lo permita, debe considerarse la posibilidad del enfoque multimodal combinando cirugía radical con inmunoterapia posterior o inhibidores de la angiogénesis 24,28-32. Se cree que la respuesta al tratamiento adyuvante es más favorable en pacientes con nefrectomia previa, buen estado general, asintomáticos y un único lugar metastático ${ }^{24}$.

\section{CONCLUSIONES}

El carcinoma de células renales con invasión hepática directa es un hallazgo infrecuente $(<10 \%$ de los CCR) y representa un signo de mal pronóstico con supervivencia a 3 años menor del $5 \%$.

Otros factores de mal pronóstico son la afectación de ganglios linfáticos, el tamaño tumoral, grado nuclear y la diferenciación sarcomatoide.

En su diagnóstico el TAC y RMN son importantes para la estadificación preoperatoria y la planificación quirúrgica.

En ausencia de tratamientos alternativos efectivos, la nefrectomía radical con hepatectomía parcial en bloque representa el único tratamiento potencialmente curativo y está justificada en pacientes seleccionados.

\section{REFERENCIAS}

1. Wong JA, Whelan T, Morse M. Radical nephrectomy with en bloc resection of liver, diaphragm, and lung for locally invasive sarcomatoid renal cell carcinoma. Urology. 2006;68 (4): 890.e1-4.

2. Bennet BC, Selby R, Bahnson RR. Surgical resection for management of renal cancer with hepatic involvement. J Urol. 1995;154(3):972-974.

3. Saitoh H. Distant metastasis of renal adenocarcinoma. Cancer. 1981 Sep 15;48(6):1487-1491.

4. Alves A, Adam R, Majno P, Delvart V, Azoulay D, Castaing D et al. Hepatic resection for metastatic renal tumors. Is it worthwhile?. Ann Surg Oncol. 2003;10(6):705-710.

5. Williams RD. Renal, perineal and ureteral neoplasms. Adult and Pediatric Urology. Edited by J.Y. Gillenwater, J.T.. Grayhack and S.S. Howards. Chicago:Yearbook Medical Publishers. 1987;(1):513-554.

6. Johnin K, Nakai O, Kataoka A, Koizumi S, Dok An C, Okada $\mathrm{Y}$ et al. Surgical management of renal cell carcinoma invading into the liver:radical nephrectomy en bloc with right hepatic lateral sector. Urology. 2001;57 (5):975.

7. Novick AC, Campbell SC. Tumores renales. In Walsh PP, Retik A.B, Vaughan ED, Wein A.J editors. Campbell. Urología. $8^{\mathrm{a}}$ ed., Buenos Aires, Editorial Médica Panamericana. 2004;2911-2979.
8. Atzpodien J, Schmitt E, Gertenbach U, Fornara P, Heynemann H, Maskow A et al. Adjuvant treatment with interleukin-2- and interferon-alpha2a-based chemoimmunotherapy in renal cell carcinoma post tumour nephrectomy: results of a prospectively randomised trial of the German Cooperative Renal Carcinoma Chemoimmunotherapy Group (DGCIN). British Journal of Cancer. 2005; 92(5): 843-846.

9. Johnson CD, Dunnick NR, Cohan RH, Illescas FF. Renal adenocarcinoma: CT staging of 100 tumors. AJR. 1987; 148(1):59-63.

10. Dekernion JB, Ramming KP, Smith RB. The natural history of metastatic renal cell carcinoma:a computer analysis. J Urol. 1978;120(2):148-152.

11. Skinner DG, Colvin RB, Vermillion CD. Diagnosis and management of renal cell carcinoma:a clinical and pathologicaln study of 309 cases. Cancer. 1971;28(5):1165-1177.

12. Hollans JM. Proceedings:cancer of the kidney-natural history and staging. Cancer. 1973;32(5):1030-1042.

13. Neves RJ, Zincke H, Taylor WF. Metastatic renal cell cancer and radical nephrectomy:identification of prognostic factors and patient survival. J Urol. 1988;139(6):11731176.

14. Stief CG, Jáhne J, Hagemann JH, Kuczyk M, Jonas U. Surgery for metachronous solitary liver metastases of renal cell carcinoma. J Urol. 1997;158(2):357-377.

15. Patiutko IuI, Matveev VB, Matveev BP, Podluzhny? DV. Liver resection in locally-spread and metastatic kidney cancer., et al:. Urologiia. 2002;(2):7-11

16. Giuliani L, Giberti C, Martorana G, Rovida S. Radical extensive surgery for renal cell carcinoma: long-term results and prognostic factors. J Urol. 1990 Mar; 143(3): 468-73; discussion 473-4.

17. Leibovich BC, Cheville JC, Lohse CM, Zincke H, Frank I, Kwon ED et al. A scoring algorithm to predict survival for patients with metastatic clear cell renal cell carcinoma:a stratification tool for prospective clinical trials. J Urol. 2005 Nov; 174(5):1759-1763; discussion 1763.

18. De Kernion JB, Sarna G, Figlin R, Lindner A, Smith RB. The treatment of renal cell carcinoma with human leukocyte alpha-interferon. J Urol. 1983;130(6):1063-1066.

19. Rosenberg SA, Lotze MT, Yang JC, Aebersold PM, Linehan WM, Seipp CA et al. Experience with the use of high-dose interleukin-2 in the treatment of 652 cancer patients. Ann Surg. 1989 Oct;210(4):474-84; discussion 484-485.

20. Foster JH. Surgical treatment for metastatic liver tumors. Hepatogastroenterology. 1990 Apr;37(2):182-187.

21. Kawata N, Hirakata H, Yuge H, Kodama M, Sugimoto S, Yagasaki $\mathrm{H}$ et al. Cytoreductive surgery with liver-involved renal cell carcinoma. Int J Urol. 2000;7(10):382-385.

22. Lang H, Nussbaum KT, Weimann A, Raab R. Liver resection for non-colorectal, non-neuroendocrine hepatic metastases. Chirurg. 1999 Apr;70(4):439-446.

23. Sakaguchi S, Hishiki S, Nakamura S, Koyano K, Kosaka A. Extension incision for renal carcinoma including invaded vena cava and right lobe of liver. Urology. 1992;39(3):285-288.

24. Van Herpen, De Mulder. Prognostic and predictive factors of immunotherapy in metastatic renal cell carcinoma. Crit Rev Oncol Hematol. 2002 Mar;41(3):327-334

25. Atzpodien J, Kirchner H, Duensing S, Lopez Hänninen E, Franzke A, Buer $J$ et al. Biochemotherapy of advanced metastatic renal-cell carcinoma:results of the combination of interleukin-2, alpha-interferon, 5-fluorouracil, vinblastine, and 13-cis-retinoic acid. World J Urol. 1995;13(3): 174-177. 
26. Atzpodien J, Kirchner H, Illiger HJ, Metzner B, Ukena D, Schott $\mathrm{H}$ et al. IL-2 in combination with IFN- alpha and 5FU versus tamoxifen in metastatic renal cell carcinoma: long-term results of a controlled randomized clinical trial. Br J Cancer. 2001;85(8):1130-1136.

27. Atzpodien J, Kirchner H, Jonas U, Bergmann L, Schott H, Heynemann $\mathrm{H}$ et al. Interleukin-2 and interferon-alpha2abased immuno-chemotherapy in advanced renal cell carcinoma:results of a prospectively randomized Trial of The German Cooperative Renal Carcinoma Chemoimmunotherapy Group (DGCIN). J Clin Oncol. 2004;22(7):1188-1194.

28. Pegram M, George D, Miller K Current status and future of oral tyrosine kinase inhibitors in the treatment of cancer (part 3 of a 3-part series on angiogenesis inhibition in solid tumor malignancies). Clin Adv Hematol Oncol. 2006;4 (12 suppl 24):1-12.

29. Feldman DR, Motzer RJ. Novel targets and therapies for metastatic renal cell carcinoma. Oncology. 2006;20(14): 1745-1753.
30. Motzer RJ, Bukowski RM. Targeted therapy for metastatic renal cell carcinoma. J Clin Oncol. 2006;24(35):5601-8.

31. García JA, Rini BI. Recent progress in the management of advanced renal cell carcinoma. CA Cancer J Clin. 2007;57:112-125.

32. Motzer RJ, Hutson TE, Tomczak P, Michaelson MD, Bukowski RM, Rixe O et al. Sunitinib versus interferon alfa in metastatic renal-cell carcinoma. The New England Journal of Medicine. 2007;356(2):115-124.

Correspondencia autora: Dra. C. Quicios Dorado Servicio de Urología. Hospital Universitario Ramón y Cajal Ctra. de Colmenar Viejo, Km 9,100 - 28034 Madrid. Tel.: 913368000

Email autora: cquicios@yahoo.es Información artículo: Nota clínica Trabajo recibido: abril 2006

Trabajo aceptado: mayo 2006 